\title{
Quantum cavitation in liquid helium
}

\author{
Montserrat Guilleumas \\ Dipartimento di Fisica, Università di Trento, 38050 Povo, Italy \\ Manuel Barranco \\ Departament d'Estructura i Constituents de la Matèria, Facultat de Física, Universitat de Barcelona, E-08028 Barcelona, Spain \\ Dora M. Jezek \\ Departamento de Física, Facultad de Ciencias Exactas y Naturales, Universidad de Buenos Aires, RA-1428 Buenos Aires, Argentina \\ Roland J. Lombard \\ Institut de Physique Nucléaire, Division de Physique Théorique. 91406 Orsay, France \\ Martí Pi \\ Departament d'Estructura i Constituents de la Matèria, Facultat de Física, Universitat de Barcelona, E-08028 Barcelona, Spain
}

(Received 5 January 1996; revised manuscript received 29 July 1996)

\begin{abstract}
Using a functional-integral approach, we have determined the temperature below which cavitation in liquid helium is driven by thermally assisted quantum tunneling. For both helium isotopes, we have obtained the crossover temperature in the whole range of allowed negative pressures. Our results are compatible with recent experimental results on ${ }^{4} \mathrm{He}$. [S0163-1829(96)01946-7]
\end{abstract}

The possibility of having observed quantum cavitation in superfluid ${ }^{4} \mathrm{He}$ has been put forward by Balibar et al. ${ }^{1}$ These authors have used a hemispherical transducer that focuses a sound wave in a small region of a cell where cavitation is induced in liquid ${ }^{4} \mathrm{He}$ at low temperature. The analysis of their experimental data is complicated by the fact that neither the pressure $(P)$ nor the temperature $(T)$ at the focus can be directly measured. This makes the determination of the thermal-to-quantum cavitation crossover temperature $T^{*}$ depend on the theoretical equation of state (EOS) near the spinodal point. Using the results of Ref. 2, they conclude that $T^{*} \sim 200 \mathrm{mK}$, in agreement with the prediction of Ref. 2. However, using for instance the EOS of Ref. 3, which reproduces the spinodal point microscopically calculated by Boronat et al., ",5 the "experimental", result becomes $120 \mathrm{mK}$.

The first detailed description of the cavitation process in liquid helium was provided by Lifshitz and Kagan, ${ }^{6}$ who used the classical capillarity model near the saturation line, and a density functional-like description near the spinodal line. More recently, the method has been further elaborated by Xiong and Maris. ${ }^{7}$ These authors conclude that there is no clear way to interpolate between these two regimes, which makes quite uncertain the range of pressures in which each of them is valid.

In this work, we determine $T^{*}$ for ${ }^{3} \mathrm{He}$ and ${ }^{4} \mathrm{He}$ using a functional-integral approach (FIA) in conjunction with a density functional description of liquid helium. The method overcomes the conceptual limitations of previous works based on the application of zero-temperature multidimensional WKB methods, ${ }^{2}$ and the technical ones inherent to the use of parametrized bubble density profiles, ${ }^{8}$ thus putting on firmer grounds the theoretical results. Moreover, it gives $T^{*}$ in the whole pressure range.
Thermally assisted quantum tunneling is nowadays well understood (see for example Ref. 9 and references therein). Let us simply recall that at high temperatures, the cavitation rate, i.e., the number of bubbles formed per unit time and volume, is given by

$$
J_{T}=J_{0 T} e^{-\Delta \Omega_{\max } / T},
$$

where $\Delta \Omega_{\max }$ is the barrier height for thermal activation and $J_{0 T}$ is a prefactor which depends on the dynamics of the cavitation process. At low $T$, it becomes

$$
J_{Q}=J_{0 Q} e^{-S_{\min }}
$$

where $S_{\min }$ is the minimum of the imaginary-time action

$$
S(T)=\oint d \tau \int d \vec{r} \mathcal{L},
$$

$\mathcal{L}$ being the imaginary-time classical Lagrangian density of the system and the time integration is extended over a period in the potential well obtained by inverting the potential barrier. These equations hold provided the rate can be calculated in the semiclassical limit, i.e., $S_{\min } \gg 1$, which is the present case. For a given value of $T$, one has to obtain periodic solutions to the variational problem embodied in Eq. (3). Among these many periodic solutions, called thermons in Ref. 9, those relevant for the problem of finding $T^{*}$ are the ones corresponding to small oscillations around the minimum of the potential, which has an energy equal to $-\Delta \Omega_{\max }$. If $\omega_{p}$ is the angular frequency of this oscillation, $T^{*}=\hbar \omega_{p} / 2 \pi$. It is worth realizing that contrary to WKB, this procedure permits one to go continuously from one regime to the other: at $T^{*}$, Eqs. (1) and (2) coincide, whereas the WKB approach forces a zero-temperature barrier penetrability to equal a finite-temperature Arrhenius factor. ${ }^{2,8}$ 
Whether this is justified or not can only be ascertained $a$ posteriori comparing the WKB with FIA results.

To obtain the Lagrangian density $\mathcal{L}$ we have resorted to a zero-temperature density functional description of the system. ${ }^{3,10}$ This is justified in view of the low $T$ that are expected to come into play $(\leqslant 200 \mathrm{mK})$. The critical cavity density profile $\rho_{0}(r)$ is obtained solving the Euler-Lagrange equation ${ }^{7,11}$

$$
\frac{\delta \omega}{\delta \rho}=0,
$$

where $\omega(\rho)$ is the grand potential density and $\rho$ is the particle density. $\Delta \Omega_{\max }$ is given by

$$
\Delta \Omega_{\max }=\int d \vec{r}\left[\omega\left(\rho_{0}\right)-\omega\left(\rho_{m}\right)\right],
$$

where $\rho_{m}$ is the density of the metastable homogeneous liquid. It is now simple to describe the dynamics of the cavitation process in the inverted barrier well, whose equilibrium configuration corresponds to $\rho_{0}(r)$ and has an energy $-\Delta \Omega_{\max }$. We suppose that the collective velocity of the fluid associated with the bubble growth is irrotational. This is not a severe restriction since one expects only radial displacements (spherically symmetric bubbles). Introducing the velocity potential field $s(\vec{r}, t)$, we have

$$
\mathcal{L}=m \dot{\rho} s-\mathcal{H}(\rho, s),
$$

where $\mathcal{H}(\rho, s)$ is the imaginary-time Hamiltonian density. Defining $\vec{u}(\vec{r}, t) \equiv \nabla s(\vec{r}, t)$,

$$
\mathcal{H}=\frac{1}{2} m \rho \vec{u}^{2}-\left[\omega(\rho)-\omega\left(\rho_{m}\right)\right] .
$$

Hamilton's equations yield

$$
\begin{gathered}
m \dot{\rho}=\frac{\delta \mathcal{H}}{\delta s}=-m \nabla(\rho \vec{u}), \\
m \dot{s}=-\frac{\delta \mathcal{H}}{\delta \rho} .
\end{gathered}
$$

Equation (8) is the continuity equation. Taking the gradient of Eq. (9) we get

$$
m \frac{d \vec{u}}{d t}=-\nabla\left\{\frac{1}{2} m \vec{u}^{2}-\frac{\delta \omega}{\delta \rho}\right\} .
$$

Thermons $\rho(\vec{r}, t)$ are periodic solutions of Eqs. (8) and (10). From Eq. (3) and using Eqs. (6) and (8) we can write

$$
S_{\min }(T)=\oint d \tau \int d \vec{r}\left\{\frac{1}{2} m \rho \vec{u}^{2}+\omega(\rho)-\omega\left(\rho_{m}\right)\right\} .
$$

Within this model, to exactly obtain $T^{*}$ only a linearized version of Eqs. (8) and (10) around $\rho_{0}(r)$ is needed. Defining the $T^{*}$ thermon as

$$
\rho(r, t) \equiv \rho_{0}(r)+\rho_{1}(r) e^{i \omega_{p} t},
$$
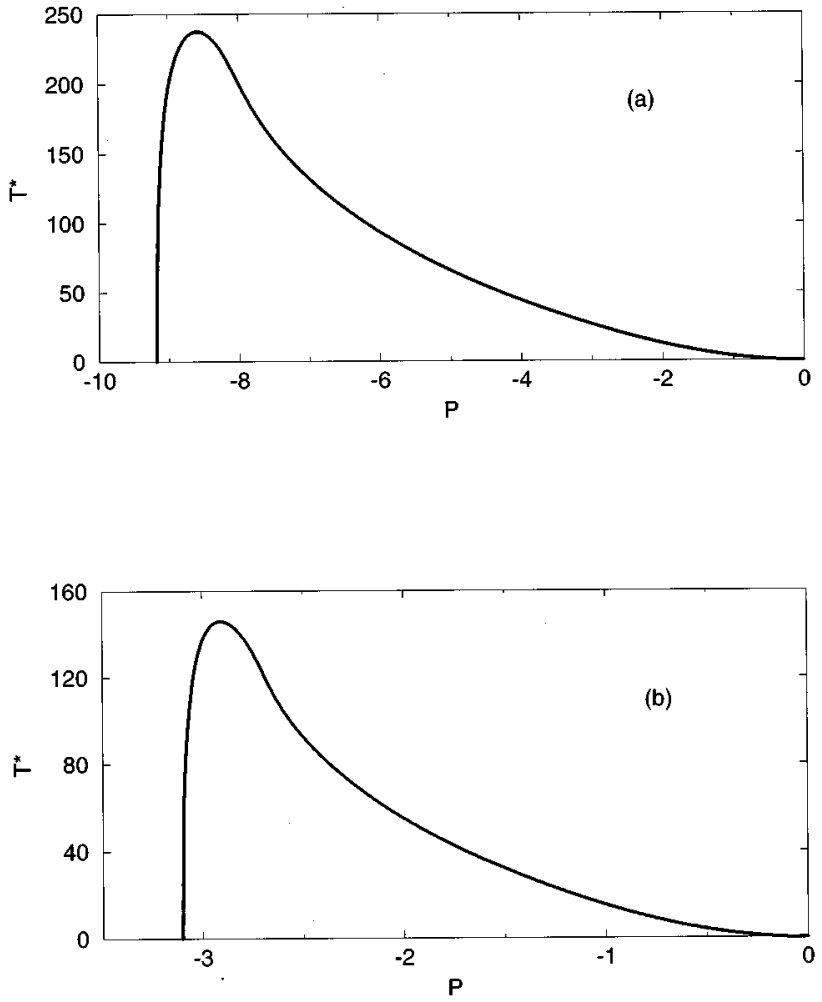

FIG. 1. (a) $T^{*}(\mathrm{mK})$ as a function of $P\left(\right.$ bar) for ${ }^{4} \mathrm{He}$. (b) Same as (a) for ${ }^{3} \mathrm{He}$.

where $\rho_{1}(r)$ is much smaller than $\rho_{0}(r)$, and keeping only first-order terms in $\vec{u}(r, t)$ and $\rho_{1}(r)$, we get

$$
m \omega_{p}^{2} \rho_{1}(r)=\nabla\left[\rho_{0}(r) \nabla\left(\frac{\delta^{2} \omega}{\delta \rho^{2}} \cdot \rho_{1}(r)\right)\right] .
$$

Here, $\left(\delta^{2} \omega / \delta \rho^{2}\right) \cdot \rho_{1}(r)$ means that $\delta \omega / \delta \rho$ has to be linearized, keeping only terms in $\rho_{1}(r)$ and its derivatives.

Equation (13) is a fourth-order linear differential, eigenvalue equation. A careful analysis shows that its physical solutions have to fulfill $\rho_{1}^{\prime}(0)=\rho_{1}^{\prime \prime \prime}(0)=0$, and fall exponentially to zero at large distances. The linearized continuity equation $\rho_{1}(r) \propto-\nabla\left(\rho_{0} \vec{u}\right)$ imposes the integral of $\rho_{1}(r)$ to yield zero when taken over the whole space.

We have solved Eq. (13) using seven point Lagrange formulas to discretize the $r$ derivatives together with a standard diagonalization subroutine. The sensibility of the solution to the precise value of the $r$ step has been carefully checked, and in most cases a value $\Delta r=0.25 \AA$ has been used.

For all pressures, only one positive $m \omega_{p}^{2}$ eigenvalue has been found. Figures $1(\mathrm{a})$ and $1(\mathrm{~b})$ show $T^{*}(\mathrm{mK})$ as a function of $P\left(\right.$ bar) for ${ }^{4} \mathrm{He}$ and ${ }^{3} \mathrm{He}$, respectively. In the case of ${ }^{4} \mathrm{He}$, the maximum $T^{*}$ is $238 \mathrm{mK}$ at $-8.58 \mathrm{bar}$, and for ${ }^{3} \mathrm{He}$ it is $146 \mathrm{mK}$ at $-2.91 \mathrm{bar}$. It is worth noting that $T^{*}$ is strongly dependent on $P$ in the spinodal region, falling to zero at the spinodal point (see also Ref. 7).

We display in Fig. 2 the $\rho_{1}(r)$ component of the thermon (12) in the case of ${ }^{4} \mathrm{He}$ (a similar figure could be drawn for ${ }^{3} \mathrm{He}$ ). For large bubbles, $\rho_{1}(r)$ is localized at the surface: the thermon is a well-defined surface excitation. It justifies the use of the capillarity approximation near saturation, or more 


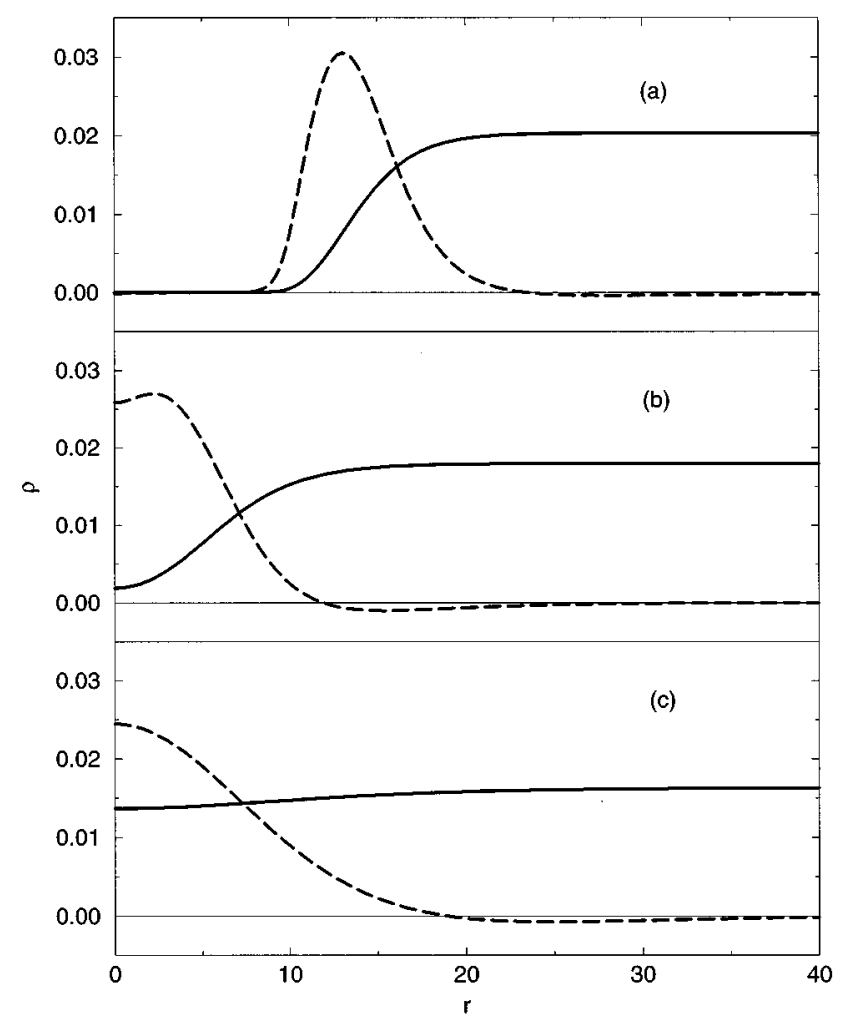

FIG. 2. Referring to ${ }^{4} \mathrm{He}$, we show (a) the particle density profile $\rho_{0}(r)$ (solid line) and the density $\rho_{1}(r)$ (dashed line) for $P=-4.59$ bar. (b) Same as (a) for $P=-8.35$ bar. (c) Same as (a) for $P=-9.16$ bar. $\rho_{1}(r)$ is drawn in arbitrary units, $\rho_{0}(r)$ in $\AA^{-3}$, and $r$ in $\AA$.

elaborate approaches, like that of Ref. 8, that consists in a simplified one-dimensional model in which the oscillations are just described by rigid displacements of the critical bubble surface.

When the density inside the bubble becomes sizable, a mixed surface-volume thermon develops, which eventually becomes a pure volume mode in the spinodal region. This mode can no longer be described as a rigid density displacement, and the above-mentioned models fail: the exact $T^{*}$ is higher than the prediction of the rigid surface displacement model because volume modes involve higher frequencies.

To determine which of the $T^{*}(P)$ shown in Fig. 1 corresponds to the actual experimental conditions, we have calculated the homogeneous cavitation pressure $P_{h} \cdot{ }^{7,11}$ It is the one the system can sustain before bubbles nucleate at an appreciable rate. We have solved the equation

$$
1=(V t)_{e} J
$$

taking $J=J_{T}$ and

$$
J_{0 T}=\frac{k_{B} T}{h V_{0}} .
$$

$V_{0}=4 \pi R_{c}^{3} / 3$ represents the volume of the critical bubble, for which we have taken $R_{c}=10 \AA$. For $T<T^{*}, J_{T}$ has to be replaced by $J_{Q}$. Lacking a better choice, we have taken $J_{0 Q}=J_{0 T}\left(T=T^{*}\right)$, and for the experimental factor $(V t)_{e}$ (experimental volume $\times$ time), two values at the limits of the
TABLE I. Crossover temperatures for two different values of the experimental volume times time.

\begin{tabular}{lcc}
\hline \hline$(V t)_{e}\left(\AA^{3} \mathrm{~s}\right)$ & ${ }^{3} \mathrm{He}$ & $T^{*}(\mathrm{mK})$ \\
& ${ }^{4} \mathrm{He}$ \\
\hline $10^{14}$ & 143 & 238 \\
$10^{4}$ & 106 & 198 \\
\hline \hline
\end{tabular}

experimental range, ${ }^{1,2}$ namely, $10^{14}$ and $10^{4} \AA^{3}$ s. For ${ }^{4} \mathrm{He}$ it yields $P_{h}=-8.57$ and -8.99 bar, respectively. The corresponding values for ${ }^{3} \mathrm{He}$ are -2.97 and -3.06 bar. This means that for both isotopes $P_{h}$ is close to the spinodal pressure. Table I displays the associated $T^{*}$ values.

The crossover temperatures are similar to those given in Ref. 2, although different functionals have been used in both calculations. As a matter of fact, this is irrelevant, since both functionals reproduce equally well the experimental quantities pertinent to the description of the cavitation process.

An explanation for the agreement between these calculations can be found in Ref. 8. In that work, using a simplified one-dimensional model in which the oscillations were modeled by rigid displacements of the bubble surface, the cavitation process was described within FIA from $T=0$ to the thermal regime. It was shown that thermally assisted quantum cavitation only adds small corrections to the $T=0$ " "instanton', solution (formally equivalent to WKB if $S_{\min } \gg 1$ ) in the quantum-to-thermal transition region.

Let us recall that the formalism used in Ref. 2 to describe quantum cavitation is a multidimensional WKB one, appropriated for a $T=0$, pure quantum state with a well-defined energy value. This approximation is well known to fail for energies close to the top of the barrier. On the contrary, the FIA here adopted deals with thermally mixed quantum states, making it possible to smoothly connect quantum and thermal regimes. ${ }^{9}$ Besides, it is technically complicated to obtain the $E=0$ instanton solution to Eqs. (8) and (10) without using some numerical approximations ${ }^{2}$ that might be unworkable in more complex physical situations, like that of a ${ }^{3} \mathrm{He}-{ }^{4} \mathrm{He}$ liquid mixture. We also want to stress again that to determine the quantity of experimental significance, namely $T^{*}$, only the thermon solution of the much simpler eigenvalue Eq. (13) is required.

To conclude, within density functional theory, we have performed a thorough description of the quantum-to-thermal transition in the process of cavitation in liquid helium based on the functional-integral approach. Our quantitative results (see also Ref. 2) indicate that the crossover temperature is below $240 \mathrm{mK}$ for ${ }^{4} \mathrm{He}$, and below $150 \mathrm{mK}$ for ${ }^{3} \mathrm{He}$. The experiments on ${ }^{4} \mathrm{He}$ yield results which, depending on which equation of state is used, are in the 120-200 mK range. Given the present uncertainties in theoretical and experimental results as well, we consider the agreement as satisfactory.

We would like to thank Sebastien Balibar, Eugene Chudnovsky, and Jacques Treiner for useful discussions. This work was supported by DGICYT (Spain) Grant No. PB920761, by the Generalitat de Catalunya Grant No. GRQ941022, by the CONICET (Argentine) Grant No. PID 97/93, and by the IN2P3-CICYT agreement. 
${ }^{1}$ S. Balibar, C. Guthmann, H. Lambare, P. Roche, E. Rolley, and H.J. Maris, J. Low Temp. Phys. 101, 271 (1995).

${ }^{2}$ H.J. Maris, J. Low Temp. Phys. 98, 403 (1995).

${ }^{3}$ A. Guirao, M. Centelles, M. Barranco, M. Pi, A. Polls, and X. Viñas, J. Phys.: Condens. Matter 4, 667 (1992)

${ }^{4}$ J. Boronat, J. Casulleras, and J. Navarro, Phys. Rev. B 50, 3427 (1994).

${ }^{5}$ The result for the spinodal pressure of Ref. 3 is $P=-9.08 \mathrm{~atm}$ at $\rho=0.0159 \AA^{-3}$ as compared with that of Ref. 4, $P=-9.30 \pm 0.15$ atm at $\rho=0.0158 \pm 0.0001 \AA^{-3}$.
${ }^{6}$ I.M. Lifshitz and Yu. Kagan, Zh. Éksp. Teor. Fiz. 62, 385 (1972) [Sov. Phys. JETP 35, 206 (1972)].

${ }^{7}$ Q. Xiong and H.J. Maris, J. Low Temp. Phys. 77, 347 (1989).

${ }^{8}$ M. Guilleumas, Ph.D. thesis, University of Barcelona, 1995 (unpublished).

${ }^{9}$ E.M. Chudnovsky, Phys. Rev. A 46, 8011 (1992).

${ }^{10}$ M. Guilleumas, F. Garcias, M. Barranco, M. Pi, and E. Suraud, Z. Phys. D 25, 227 (1993).

${ }^{11}$ D.M. Jezek, M. Guilleumas, M. Pi, M. Barranco, and J. Navarro, Phys. Rev. B 48, 16582 (1993). 